\title{
RECONSTRUCTION ALGORITHMS IN IRREGULAR SAMPLING
}

\author{
KARLHEINZ GRÖCHENIG
}

\begin{abstract}
A constructive solution of the irregular sampling problem for bandlimited functions is given. We show how a band-limited function can be completely reconstructed from any random sampling set whose density is higher than the Nyquist rate, and give precise estimates for the speed of convergence of this iteration method. Variations of this algorithm allow for irregular sampling with derivatives, reconstruction of band-limited functions from local averages, and irregular sampling of multivariate band-limited functions.
\end{abstract}

In the irregular sampling problem one is asked whether and how a bandlimited function $f$ can be completely reconstructed from its irregularly sampled values $f\left(x_{i}\right)$. This has many applications in signal and image processing, seismology, meteorology, medical imaging, etc. Finding constructive solutions of this problem has received considerable attention among mathematicians and engineers.

The mathematical literature provides several uniqueness results $[1,2,17,18$, 19]. It is now part of the folklore that for stable sampling the sampling rate must be at least the Nyquist rate [18]. These results, as deep as they are, have had little impact for the applied sciences, because they were not constructive.

If the sampling set is just a perturbation of the regular oversampling, then a reconstruction method has been obtained in a seminal paper by Duffin and Schaeffer [6] (see also [29]): if for some $L \geq 0, \alpha>0$, and $\delta>0$ the sampling points $x_{k}, k \in Z$, satisfy (a) $\left|x_{k}-\delta k\right| \leq L$ and (b) $\left|x_{k}-x_{l}\right| \geq \alpha, k \neq l$, then the norm equivalence $A \int_{R}|f(x)|^{2} d x \leq \sum_{k}\left|f\left(x_{k}\right)\right|^{2} \leq B \int_{R}|f(x)|^{2} d x$ holds for all $f \in L^{2}(R)$ such that supp $\widehat{f} \subseteq[-\omega, \omega]$ with $\omega<\pi / \delta$. This norm equivalence implies that it is possible to reconstruct $f$ through an iterative procedure, the so-called frame method. Most of the later work on constructive methods consists of variations of this method [3,21,22, 26].

The above conditions on the sampling set exclude random irregular sampling sets, e.g., sets with regions of higher sampling density. A partial, but undesirable remedy, to handle highly irregular sampling sets, would be to force the above conditions by throwing away information on part of the points and accept a very slow convergence of the iteration.

Received by the editor August 14, 1990 and, in revised form, February 26, 1991.

1991 Mathematics Subject Classification. Primary 11L99, 65D99, 42A65, 42B99.

Key words and phrases. Band-limited functions, irregular sampling, Wirtinger's inequality.

This work was partially supported by DARPA-grant AFOSR-90-0311. 
Since the constants $A$ and $B$ are not explicit, it is impossible to estimate the rate of convergence of the procedure, which only depends on the number $(B-A) /(B+A)$.

Recently the method of projections onto convex sets (POCS; cf. [28]) has been applied to irregular sampling problems $[21,25,27]$. The emphasis in these works is more on the interpolation of sampled data $\left(x_{i}, y_{i}\right)$ by a bandlimited function satisfying $f\left(x_{i}\right)=y_{i}$. Numerical questions such as feasible sampling densities or speed of convergence of these algorithms were not touched. Nevertheless, the ingenious method of POCS has the potential to provide a complete reconstruction of a band-limited function from irregularly sampled values.

A very general qualitative theory for irregular sampling has been developed by Feichtinger and the author in a series of papers [8, 9, 10, 11] (cf. [14] for an elementary exposition). It is based on the fact that every band-limited function $f$ satisfies a reproducing formula $f * g=f$ for a suitable function $g$. In contrast to the work mentioned so far, these algorithms work for a large class of norms and are extremely stable with respect to perturbations [12]. In numerical experiments these algorithms have performed equally well or better than other methods [13]. But again, the constants are rather implicit, and it is unclear for which sampling rates and how fast these algorithms will converge.

In all these investigations of irregular sampling the numerical aspects have been rather neglected. It is quite easy to implement sampling geometries for which the iteration diverges. Thus, lacking precise estimates of the constants involved, numerical work had to be done on a trial-and-error basis. For effective applications of an iterative reconstruction algorithm one must answer the following questions:

1. For which sampling densities does the iteration converge to the original function $f$ ?

2. How fast will these iterations converge to the original signal? How many iterations have to be computed to achieve a certain accuracy?

This paper aims at the quantitative aspects of the irregular sampling problem. In this paper these questions will be answered for several different reconstruction methods. Breaking a habit, only Hilbert spaces of band-limited functions are considered.

In $\S 2$ an iterative method is developed which yields a complete reconstruction of a band-limited function from any randomly distributed sampling set with a density higher than the Nyquist density (Theorem 1). An essential part of this theorem is a precise estimate for the rate of convergence of this algorithm. Section 3 shows that a band-limited function can even be reconstructed completely from local averages around the sampling points $x_{i}$ instead of the precise values $f\left(x_{i}\right)$.

Section 4 contains some variations to show the potential of this method. The speed of convergence can be increased considerably by (a) using piecewise linear approximations, or (b) irregular sampling with derivatives. Finally a multivariate version for irregular sampling of band-limited functions on $R^{n}$ is proven.

The underlying idea is to view a Hilbert space of band-limited functions with fixed spectrum as a reproducing kernel Hilbert space and then approximate a band-limited function $f$ by a discretized version $A f$ which contains the sampled values of $f$ only. The reconstruction of $f$ is then based on a Neumann 
series expansion of $A^{-1}$. The novelty of this paper lies in explicit and sharp estimates of the error $f-A f$.

For an overview of the extensive literature on irregular sampling and references to other facets of this problem, we refer to [20] and the long lists of references in $[9,10]$.

\section{Preliminaries}

As usual, $L^{2}(R)$ denotes the Hilbert space of square-integrable functions on $R$ with norm $\|f\|=\left(\int_{-\infty}^{\infty}|f(x)|^{2} d x\right)^{1 / 2}$.

Let $\Omega$ be a compact set and let $\omega=\sup _{t \in \Omega}|t|$ be the extension of $\Omega$. Then $B^{2}(\Omega)=\left\{f \in L^{2}(R): \operatorname{supp} \hat{f} \subseteq \Omega\right\}$ denotes the closed subspace of squareintegrable band-limited functions with spectrum (= support of the Fourier transform) in $\Omega$. By $P$ we denote the orthogonal projection from $L^{2}(R)$ onto $B^{2}(\Omega)$, defined by $(P f)^{\wedge}=\chi_{\Omega} \cdot \widehat{f}$ a.e., where $\chi_{\Omega}$ is the characteristic function of the set $\Omega$. Here the Fourier transform is defined by $\widehat{f}(\xi)=\int_{R} f(x) e^{-i x \xi} d x$.

A sequence $X=\left(x_{i}\right)_{i \in Z}, \cdots<x_{i-1}<x_{i}<x_{i+1}<\cdots$, is $\delta$-dense, if $\sup _{i}\left(x_{i+1}-x_{i}\right) \leq \delta$. It is not required that the $x_{i}$ 's are separated from each other by a minimal distance. The inverse $\delta^{-1}$ is usually referred to as the sampling rate; thus a smaller value of $\delta$ corresponds to a higher sampling rate.

Lemma 1 (Wirtinger's inequality). (a) If $f, f^{\prime} \in L^{2}(a, b)$ and either $f(a)=0$ or $f(b)=0$, then

$$
\int_{a}^{b}|f(x)|^{2} d x \leq \frac{4}{\pi^{2}}(b-a)^{2} \int_{a}^{b}\left|f^{\prime}(x)\right|^{2} d x .
$$

(b) If $f, f^{\prime \prime} \in L^{2}(a, b)$ and $f(a)=f(b)=0$, then

$$
\int_{a}^{b}|f(x)|^{2} d x \leq\left(\frac{b-a}{\pi}\right)^{4} \int_{a}^{b}\left|f^{\prime \prime}(x)\right|^{2} d x .
$$

Part (a) of the lemma follows from [15, p. 184], by a change of variables, part (b) occurs first in [7], see also [5].

Lemma 2 (Bernstein's inequality). If $f \in B^{2}(\Omega)$ and $\omega=\sup _{t \in \Omega}|t|$, then $f^{\prime} \in B^{2}(\Omega)$ and

$$
\left\|f^{\prime}\right\| \leq \omega\|f\| .
$$

(Here the normalization of the Fourier transform is crucial!)

Lemma 3 (Iterative reconstruction). Let $A$ be a bounded operator on a Banach space $B$ such that $\|\mathrm{Id}-A\|^{\prime}<1$, where $\|\cdot\|^{\prime}$ denotes the operator norm on $B$. Then $A$ is invertible on $B$ and $A^{-1}=\sum_{n=0}^{\infty}(\mathrm{Id}-A)^{n}$.

(a) Moreover, every $f \in B$ can be reconstructed by the iteration

$$
\phi_{0}=A f, \quad \phi_{n+1}=\phi_{n}-A \phi_{n}, \quad f=\sum_{n=0}^{\infty} \phi_{n}
$$

with convergence in $B$.

(b) Setting $f_{n}=\sum_{k=0}^{n} \phi_{k}$, the error is

$$
\left\|f-f_{n}\right\|_{B} \leq\|\mathrm{Id}-A\|^{\prime n+1} \frac{1+\|\mathrm{Id}-A\|^{\prime}}{1-\|\mathrm{Id}-A\|^{\prime}}\|f\|_{B} .
$$


Proof. The Neumann series for the inverse of an operator is a standard fact. Part (a) then follows from

$$
f=A^{-1} A f=\sum_{n=0}^{\infty}(\mathrm{Id}-A)^{n} A f
$$

by setting $\phi_{0}=A f$ and

$$
\phi_{n}=(\operatorname{Id}-A)^{n} A f=(\operatorname{Id}-A)(\operatorname{Id}-A)^{n-1} A f=\phi_{n-1}-A \phi_{n-1} .
$$

For (b) we observe that

$$
f-f_{n}=\sum_{k=n+1}^{\infty} \phi_{k}=\sum_{k=n+1}^{\infty}(\mathrm{Id}-A)^{k} A f .
$$

The error estimate is now a consequence of

$$
\begin{aligned}
\left\|\sum_{k=n+1}^{\infty}(\mathrm{Id}-A)^{k} A\right\|^{\prime} & \leq \sum_{k=n+1}^{\infty}\|\mathrm{Id}-A\|^{\prime k}\|A\|^{\prime} \\
& \leq \frac{\|\mathrm{Id}-A\|^{\prime n+1}}{1-\|\mathrm{Id}-A\|^{\prime}} \cdot\left(1+\|\mathrm{Id}-A\|^{\prime}\right) .
\end{aligned}
$$

This simple lemma is the core of many important algorithms in analysis. It reduces the design of an iterative reconstruction algorithm to finding an appropriate approximation of the identity operator.

\section{A SIMPLE RECONSTRUCTION ALGORITHM}

In this section we prove a simple iterative algorithm for the reconstruction of a band-limited function $f \in B^{2}(\Omega)$ from its randomly sampled values $f\left(x_{i}\right)$ and discuss its stability and its speed of convergence. The algorithm is but a simplification of $[9,10]$ for the $L^{2}$-situation. The main contribution here are explicit and optimal estimates.

Let $X=\left(x_{i}\right)_{i \in Z}, \cdots<x_{i-1}<x_{i}<x_{i+1}<\cdots$, be a sampling set of density $\delta$. Denote the midpoints by $y_{i}=\left(x_{i+1}+x_{i}\right) / 2$ and set $\chi_{i}=\chi_{\left[y_{i-1}, y_{i}\right)}$. Then $y_{i}-x_{i} \leq \delta / 2, x_{i}-y_{i-1} \leq \delta / 2$, and the $\chi_{i}$ 's form a partition of unity, i.e., $\sum_{i=-\infty}^{\infty} \bar{\chi}_{i}(x)=1$ for all $x$.

Theorem 1. (a) Reconstruction: If $\delta<\pi / \omega$, i.e., if the sampling rate of $X$ is higher than the Nyquist rate, then every $f \in B^{2}(\Omega)$ can be completely reconstructed from the sampling values $f\left(x_{i}\right)$ on an arbitrary $\delta$-dense sampling set $X$ by the following iteration:

$$
\begin{gathered}
\phi_{0}=P\left(\sum_{i \in Z} f\left(x_{i}\right) \chi_{i}\right), \\
\phi_{n+1}=\phi_{n}-P\left(\sum_{i \in Z} \phi_{n}\left(x_{i}\right) \chi_{i}\right),
\end{gathered}
$$

and

$$
f=\sum_{n=0}^{\infty} \phi_{n}
$$

where all sums converge in $L^{2}$. 
(b) Rate of convergence: Let $f_{n}=\sum_{k=0}^{n} \phi_{k}$ be the resulting approximation of $f$ after $n$ iterations of (11). Then

$$
\left\|f-f_{n}\right\| \leq\left(\frac{\delta \omega}{\pi}\right)^{n+1} \frac{\pi+\delta \omega}{\pi-\delta \omega}\|f\| .
$$

Proof. Define the approximation operator $A$ by

$$
A f=P\left(\sum_{i \in Z} f\left(x_{i}\right) \chi_{i}\right) .
$$

It is easily seen that $A$ is a bounded linear operator from $B^{2}(\Omega)$ into $B^{2}(\Omega)$ (see also (18) below). We have to find an estimate on $\|f-A f\|$ for $f \in B^{2}(\Omega)$. By writing $f \in B^{2}(\Omega)$ as $f=P f=P\left(\sum f \chi_{i}\right)$, one obtains

$$
\begin{aligned}
\|f-A f\|^{2} & =\left\|P\left(\sum_{i \in Z}\left(f-f\left(x_{i}\right)\right) \chi_{i}\right)\right\|^{2} \leq\left\|\sum_{i \in Z}\left(f-f\left(x_{i}\right)\right) \chi_{i}\right\| \\
& =\int_{R}\left|\sum_{i}\left(f(x)-f\left(x_{i}\right)\right) \chi_{i}\right|^{2} d x .
\end{aligned}
$$

Since the $\chi_{i}$ 's are characteristic functions and have mutually disjoint support, the last expression equals

$$
\int_{R}\left(\sum_{i}\left|f(x)-f\left(x_{i}\right)\right|^{2} \chi_{i}(x)\right) d x=\sum_{i} \int_{y_{i-1}}^{y_{i}}\left|f(x)-f\left(x_{i}\right)\right|^{2} d x .
$$

The interchange of sum and integral is justified because the sum converges absolutely.

Next one applies Wirtinger's inequality (Lemma 1(a)) to each individual term in the following way:

$$
\begin{aligned}
& \int_{y_{i-1}}^{y_{i}}\left|f(x)-f\left(x_{i}\right)\right|^{2} d x=\int_{y_{i-1}}^{x_{i}} \cdots+\int_{x_{i}}^{y_{i}} \cdots \\
& \quad \leq \frac{4}{\pi^{2}}\left(x_{i}-y_{i-1}\right)^{2} \int_{y_{i-1}}^{x_{i}}\left|f^{\prime}(x)\right|^{2} d x+\frac{4}{\pi^{2}}\left(y_{i}-x_{i}\right)^{2} \int_{x_{i}}^{y_{i}}\left|f^{\prime}(x)\right|^{2} d x \\
& \quad \leq \frac{4}{\pi^{2}}\left(\frac{\delta}{2}\right)^{2} \int_{y_{i-1}}^{y_{i}}\left|f^{\prime}(x)\right|^{2} d x .
\end{aligned}
$$

Summing over $i$ and using Bernstein's inequality, one obtains

$$
\|f-A f\|^{2} \leq \frac{\delta^{2}}{\pi^{2}} \sum_{i} \int_{y_{i-1}}^{y_{i}}\left|f^{\prime}(x)\right|^{2} d x=\frac{\delta^{2}}{\pi^{2}}\left\|f^{\prime}\right\|^{2} \leq \frac{\delta^{2} \omega^{2}}{\pi^{2}}\|f\|^{2} .
$$

Thus we have obtained the desired estimate

$$
\|f-A f\| \leq \frac{\delta \omega}{\pi}\|f\| \text { for all } f \in B^{2}(\Omega) .
$$

Consequently, for $\delta \omega / \pi<1$, Lemma 3 is applicable and the reconstruction $(10)-(12)$ and the error estimate (13) are proved. Since the start of the iteration 
is $\phi_{0}$, the reconstruction contains indeed only the information on the samples $f\left(x_{i}\right)$.

Since $A^{-1}$ is bounded on $B^{2}(\Omega)$, the stability of the reconstruction follows from $\|f\|=\left\|A^{-1} A f\right\| \leq\left\|A^{-1}\right\|^{\prime}\|P\|^{\prime}\left\|\sum_{i \in Z} f\left(x_{i}\right) \chi_{i}\right\|$ :

Corollary 1. The sampling from $X$ is stable, i.e.,

$$
\|f\| \leq C\left\|\sum_{i \in Z} f\left(x_{i}\right) \chi_{i}\right\| .
$$

If $X$ is separated, i.e., $\left|x_{i}-x_{j}\right| \geq \alpha>0$, then obviously

$$
\|f\| \leq C^{\prime}\left(\sum_{i \in Z}\left|f\left(x_{i}\right)\right|^{2}\right)^{1 / 2} .
$$

Corollary 2. Set $e_{i}=A^{-1} P \chi_{i}$. Then the $e_{i}$ 's are a bounded sequence in $B^{2}(\Omega)$ and every $f \in B^{2}(\Omega)$ has the representation

$$
f=A^{-1} P\left(\sum_{i} f\left(x_{i}\right) \chi_{i}\right)=\sum_{i} f\left(x_{i}\right) e_{i}
$$

with convergence of the partial sums in the $L^{2}$ norm.

Discussion. 1. The Nyquist rate is $\pi / \omega$; thus Theorem 1 gives indeed the optimal estimate for the sampling density. To my knowledge, the only reconstruction method that works at exactly the Nyquist rate is the cardinal series for regular sampling $[16,4]$.

2. It should be emphasized that the only condition on the sampling set $X$ is its density. In contrast to the other constructive methods, neither separation nor any other structure of $X$ is required. The convergence of the reconstruction of Duffin and Schaeffer [6] by means of the "frame operator" and all methods based on it seem to be very slow, when the sampling density varies locally. The algorithm of Theorem 1 balances the local variation of the sampling density with the partition of unity $\chi_{i}$ and thus adapts to local changes of the sampling density.

3. In the so-called sample-and-hold reconstruction the approximation operator

$$
A_{\mathrm{SH}} f=P\left(\sum_{i} f\left(x_{i}\right) \chi_{\left[x_{i}, x_{i+1}\right)}\right)
$$

is used. Repeating the same argument as above, with equation (17) replaced by $\int_{x_{i}}^{x_{i+1}}\left|f(x)-f\left(x_{i}\right)\right|^{2} d x \leq 4 \pi^{-2}\left(x_{i+1}-x_{i}\right)^{2} \int_{x_{i}}^{x_{i+1}}\left|f^{\prime}(x)\right|^{2} d x$, one arrives at

$$
\|f-A f\| \leq \frac{2 \delta \omega}{\pi}\|f\| .
$$

Thus convergence of the iteration can be guaranteed only if $\delta<\pi / 2 \omega$.

4. The factorization (6) and the representation (21) can be used as the starting point for a detailed error analysis of the algorithm. This has been done in a more general context in [12]. The arguments can easily be simplified to suit the situation of Theorem 1 and need not be repeated here. 
5. Writing $f_{1}=A f$, the iteration can also be written as

$$
f_{n+1}=T f_{n}=f_{n}+A\left(f-f_{n}\right),
$$

as is readily seen from

$$
\begin{aligned}
f_{n+1} & =\sum_{k=0}^{n+1}(\mathrm{Id}-A)^{k} A f=(\mathrm{Id}-A) \sum_{k=0}^{n}(\mathrm{Id}-A)^{k} A f+A f \\
& =f_{n}+A\left(f-f_{n}\right) .
\end{aligned}
$$

In this form it resembles the method of projections onto convex sets. In the POCS-method, however, the operators, namely projections, are forced upon the user, whereas in our approach one may choose any convenient approximation operator $A$ (see the next sections and [10]). The $A$ in Theorem 1 is certainly easier to handle than the projection onto $\left\{g \in B^{2}(\Omega), g\left(x_{i}\right)=f\left(x_{i}\right), i \in Z\right\}$, which is needed for POCS.

6. The error estimate (13) allows one to determine the number of iterations required for a certain accuracy. For instance, in order to achieve an accuracy of $\left\|f-f_{n}\right\| /\|f\|<0.001$ with twofold oversampling, i.e., $\delta \leq \pi / 2 \omega$, about 11 iterations are necessary. Since for industrial standards oversampling rates of 4 are customary, e.g., in CD players, the speed of convergence is very satisfactory.

The geometric decay of the relative error has also been verified numerically [13].

\section{COMPLETE RECONSTRUCTION FROM AVERAGES}

For physical reasons, e.g., the inertia of the measurement apparatus, it is impossible to measure the value of a signal precisely at time $x$. In practice, only a local average of $f$ near $x_{i}$ can be measured and used as the input for a reconstruction method.

To make a realistic model, we assume that the local averages are given by

$$
f_{i}=\int f(x) u_{i}(x) d x=\left\langle u_{i}, f\right\rangle,
$$

where $u_{i}, i \in Z$, is a collection of averaging functions, i.e., they satisfy the properties

$$
\operatorname{supp} u_{i} \subseteq\left[x_{i}-\delta / 2, x_{i}+\delta / 2\right], \quad 0 \leq u_{i}(x) \leq 1, \quad \int u_{i}(x) d x=1 .
$$

Observe that the averaging procedure is allowed to vary from point to point.

It is clear that from these local averages one should obtain at least a good approximation of the original function if $\delta$ is small [26]. Next we show that band-limited functions are even completely determined by their local averages:

Theorem 2. Assume that $X=\left(x_{i}\right)_{i \in Z}$ is a $\delta$-dense sampling set and that $u_{i}, i \in$ $Z$, is a collection of averaging functions with properties (26). If $\delta<(\sqrt{2} \omega)^{-1}$, then every $f \in B^{2}(\Omega)$ is uniquely determined by the local averages $\left\langle u_{i}, f\right\rangle$ around $x_{i}$. Moreover, $f$ can be reconstructed by the following iteration scheme:

$$
\phi_{0}=P\left(\sum_{i}\left\langle u_{i}, f\right\rangle \chi_{i}\right)
$$




$$
\phi_{n+1}=\phi_{n}-P\left(\sum_{i \in Z}\left\langle u_{i}, \phi_{n}\right\rangle \chi_{i}\right) \text {, }
$$

and

$$
f=\sum_{n=0}^{\infty} \phi_{n},
$$

where all sums converge in $L^{2}$.

Proof. 1. In this case we have to estimate $\left\|f-A_{u} f\right\|$ for $f=P f \in B^{2}(\Omega)$, where $A_{u}$ is the approximation operator

$$
A_{u} f=P\left(\sum_{i}\left\langle u_{i}, f\right\rangle \chi_{i}\right) .
$$

Keeping the notation of $\S 2$, one calculates as in (15) and (16):

$$
\left\|f-A_{u} f\right\|^{2} \leq \sum_{i} \int_{y_{i-1}}^{y_{i}}\left|f(x)-\left\langle u_{i}, f\right\rangle\right|^{2} d x .
$$

Since $\int u_{i}(x) d x=1, u_{i}(x) \geq 0$, each term can be estimated by

$$
\begin{aligned}
\int_{y_{i-1}}^{y_{i}} & \left|f(x)-\int_{x_{i}-\delta / 2}^{x_{i}+\delta / 2} u_{i}(y) f(y) d y\right|^{2} d x \\
& =\int_{y_{i-1}}^{y_{i}}\left|\int_{x_{i}-\delta / 2}^{x_{i}+\delta / 2}(f(x)-f(y)) u_{i}(y) d y\right|^{2} d x \\
& \leq \int_{y_{i-1}}^{y_{i}} \sup _{y:|y-x| \leq \delta}|f(x)-f(y)|^{2} d x .
\end{aligned}
$$

The integrand

$$
\operatorname{osc}_{\delta} f(x)=\sup _{y:|y-x| \leq \delta}|f(y)-f(x)|
$$

is often called the $\delta$-oscillation of $f$. It measures the local variation of $f$ near a point $x$ and has been very useful in the abstract theory of irregular sampling [10].

Summing (32) over $i$, one obtains

$$
\left\|f-A_{u} f\right\| \leq\left\|\operatorname{osc}_{\delta} f\right\| .
$$

2. A simple estimate for the oscillation is given in the following lemma.

Lemma 4. If $f^{\prime} \in L^{2}(R)$, then

$$
\left\|\operatorname{osc}_{\delta} f\right\| \leq \sqrt{2} \delta\left\|f^{\prime}\right\| .
$$

Proof of the lemma. The relation

$$
|f(y)-f(x)|=\left|\int_{x}^{y} f^{\prime}(t) d t\right| \leq \sqrt{|y-x|}\left(\int_{x}^{y}\left|f^{\prime}(t)\right|^{2} d t\right)^{1 / 2}
$$

implies

$$
\sup _{y:|y-x| \leq \delta}|f(y)-f(x)| \leq \sqrt{\delta}\left(\int_{-\delta}^{\delta}\left|f^{\prime}(x+t)\right|^{2} d t\right)^{1 / 2} .
$$


Integration over $x$ and the use of Fubini's theorem yields

$$
\begin{aligned}
\int_{-\infty}^{\infty} \operatorname{osc}_{\delta} f(x)^{2} d x & \leq \delta \int_{-\infty}^{\infty}\left(\int_{-\delta}^{\delta}\left|f^{\prime}(x+t)\right|^{2} d t\right) d x \\
& =\delta \int_{-\delta}^{\delta} \int_{-\infty}^{\infty}\left|f^{\prime}(x+t)\right|^{2} d x d t=2 \delta^{2}\left\|f^{\prime}\right\|^{2}
\end{aligned}
$$

3. For $f \in B^{2}(\Omega)$, the estimate (34) can be continued by

$$
\left\|f-A_{u} f\right\| \leq \sqrt{2} \delta\left\|f^{\prime}\right\| \leq \sqrt{2} \delta \omega\|f\| .
$$

For $\sqrt{2} \delta \omega<1$, Lemma 3 is applicable and yields the stated algorithm.

Remarks. 1. By Lemma $3(\mathrm{~b})$, the rate of convergence is of the order $O\left((\sqrt{2} \delta \omega)^{n+1}\right)$. Clearly, stability and a series expansion as in (21) can be obtained in the same way as above.

2. The precise nature of the averages $u_{i}$ is usually unknown. If one thinks of $A_{u}$ as a physical measurement apparatus or a black box with constant characteristics, then in repeated applications of $A_{u}$ (= repeated measurements) always the same average is used at $x_{i}$, and thus our ignorance about the averages should not present any problem.

\section{VARIATIONS}

In this section other reconstruction algorithms are considered. The objective of the first two subsections is to show how the rate of convergence of the algorithm can be speeded up by using a different resolution of unity or by using irregular sampling with derivatives. Although the results are not as complete as in $\S 2$, they could be very useful in many applications with high oversampling.

4.1. Piecewise linear approximations. Instead of the characteristic functions $\chi_{i}=\chi_{\left[y_{i-1}, y_{i}\right]}$ one may use other partitions of unity $[9,10]$. In this section we use the triangular functions

$$
\psi_{i}(x)= \begin{cases}\frac{x-x_{i-1}}{x_{i}-x_{i-1}} & \text { for } x_{i-1} \leq x \leq x_{i}, \\ \frac{x_{i+1}-x}{x_{i+1}-x_{i}} & \text { for } x_{i} \leq x \leq x_{i+1} \\ 0 \quad \text { elsewhere } & \end{cases}
$$

Then $\sum_{i} \psi_{i}(x)=1$ for all $x$ and

$$
\sum_{i} f\left(x_{i}\right) \psi_{i}(x)=f\left(x_{i}\right)+\frac{f\left(x_{i+1}\right)-f\left(x_{i}\right)}{x_{i+1}-x_{i}}\left(x-x_{i}\right) \text { for } x_{i} \leq x \leq x_{i+1}
$$

becomes the piecewise linear interpolation of $f$.

Theorem 3. If $\delta<\pi / \omega$, then $f \in B^{2}(\Omega)$ can be reconstructed from any $\delta$-dense sampling set $X=\left(x_{i}\right)_{i \in Z}$ by means of the iteration procedure

$$
\begin{gathered}
\phi_{0}=P\left(\sum_{i \in Z} f\left(x_{i}\right) \psi_{i}\right), \\
\phi_{n+1}=\phi_{n}-P\left(\sum_{i \in Z} \phi_{n}\left(x_{i}\right) \psi_{i}\right),
\end{gathered}
$$


and

$$
f=\sum_{n=0}^{\infty} \phi_{n}
$$

where all sums converge in $L^{2}$. If $f_{n}=\sum_{k=0}^{n} \phi_{k}$, the rate of convergence is

$$
\left\|f-f_{n}\right\| \leq\left(\frac{\delta \omega}{\pi}\right)^{2(n+1)} \frac{\pi^{2}+\delta^{2} \omega^{2}}{\pi^{2}-\delta^{2} \omega^{2}}\|f\| .
$$

Proof. By (40) we deal with the approximation operator

$$
A_{1} f(x)=P\left(\sum_{i}\left(f\left(x_{i}\right)+\frac{f\left(x_{i+1}\right)-f\left(x_{i}\right)}{x_{i+1}-x_{i}}\left(x-x_{i}\right)\right) \chi_{\left[x_{i}, x_{i+1}\right]}(x)\right) .
$$

With the abbreviation $\Delta_{i}=\left(f\left(x_{i+1}\right)-f\left(x_{i}\right)\right) /\left(x_{i+1}-x_{i}\right)$ one calculates as in (1.5) and (16):

$$
\left\|f-A_{1} f\right\|^{2} \leq \sum_{i} \int_{x_{i}}^{x_{i+1}}\left|f(x)-f\left(x_{i}\right)-\Delta_{i}\left(x-x_{i}\right)\right|^{2} d x .
$$

Since the integrand vanishes at $x_{i}$ and $x_{i+1}$, the second form of Wirtinger's inequality (2) yields

$$
\begin{aligned}
\int_{x_{i}}^{x_{i+1}} & \left|f(x)-f\left(x_{i}\right)-\Delta_{i}\left(x-x_{i}\right)\right|^{2} d x \\
& \leq\left(\frac{x_{i+1}-x_{i}}{\pi}\right)^{4} \int_{x_{i}}^{x_{i+1}}\left|f^{\prime \prime}(x)\right|^{2} d x
\end{aligned}
$$

Taking the sum over $i$, one obtains for $f \in B^{2}(\Omega)$

$$
\left\|f-A_{1} f\right\|^{2} \leq \frac{\delta^{4}}{\pi^{4}}\left\|f^{\prime \prime}\right\|^{2} \leq \frac{\delta^{4} \omega^{4}}{\pi^{4}}\|f\|^{2} .
$$

For $\delta \omega<\pi$, the algorithm is implied by Lemma 3. The error is now of the order $O\left((\delta \omega / \pi)^{2(n+1)}\right)$.

Remark. Using higher-order splines for the partition of unity, we may increase the rate of convergence. It is an open problem to determine the required sampling rate and the optimal constants for this case.

\subsection{Irregular sampling with derivatives.}

Theorem 4. Assume that the first $k$ derivatives $f, f^{\prime}, \ldots, f^{(k)}$ are sampled at $a \delta$-dense set $X=\left(x_{i}\right)_{i \in Z}$. If $\delta<\pi / \omega$, then the iteration procedure

$$
\begin{gathered}
\phi_{0}=P\left(\sum_{i \in Z}\left(\sum_{j=0}^{k} f^{(j)}\left(x_{i}\right) \frac{\left(x-x_{i}\right)^{j}}{j !}\right) \chi_{i}\right), \\
\phi_{n+1}=\phi_{n}-P\left(\sum_{i \in Z}\left(\sum_{j=0}^{k} \phi_{n}^{(j)}\left(x_{i}\right) \frac{\left(x-x_{i}\right)^{j}}{j !}\right) \chi_{i}\right),
\end{gathered}
$$


and

$$
f=\sum_{n=0}^{\infty} \phi_{n}
$$

is a complete reconstruction of $f \in B^{2}(\Omega)$. The rate of convergence is

$$
\left\|f-f_{n}\right\| \leq\left(\frac{\delta \omega}{\pi}\right)^{(k+1)(n+1)} \frac{\pi^{k+1}+(\delta \omega)^{k+1}}{\pi^{k+1}-(\delta \omega)^{k+1}}\|f\| .
$$

Proof. We set for $f \in B^{2}(\Omega)$

$$
A_{k} f(x)=P\left(\sum_{i \in Z}\left(\sum_{j=0}^{k} f^{(j)}\left(x_{i}\right) \frac{\left(x-x_{i}\right)^{j}}{j !}\right) \chi_{i}(x)\right) .
$$

Again, we only need to show that $A_{k}$ is invertible on $B^{2}(\Omega)$ and apply Lemma 3. First one calculates

$$
\left\|f-A_{k} f\right\|^{2} \leq \sum_{i} \int_{y_{i-1}}^{y_{i}}\left|f(x)-\sum_{j=0}^{k} f^{(j)}\left(x_{i}\right) \frac{\left(x-x_{i}\right)^{j}}{j !}\right|^{2} d x .
$$

Since the first $k$ derivatives of the integrand vanish at each $x_{i}$, Wirtinger's inequality is applicable $k$ times to $\int_{y_{i-1}}^{y_{i}}=\int_{y_{i-1}}^{x_{i}}+\int_{x_{i}}^{y_{i}}$, and one obtains

$$
\begin{aligned}
\int_{y_{i-1}}^{y_{i}}\left|f(x)-\sum_{j=0}^{k} f^{(j)}\left(x_{i}\right) \frac{\left(x-x_{i}\right)^{j}}{j !}\right|^{2} d x \\
\quad \leq \frac{\delta^{2}}{\pi^{2}} \int_{y_{i-1}}^{y_{i}}\left|f^{\prime}(x)-\sum_{j=1}^{k} f^{(j)}\left(x_{i}\right) \frac{\left(x-x_{i}\right)^{j-1}}{(j-1) !}\right|^{2} d x \\
\leq \frac{\delta^{4}}{\pi^{4}} \int_{y_{i-1}}^{y_{i}}\left|f^{\prime \prime}(x)-\sum_{j=2}^{k} f^{(j)}\left(x_{i}\right) \frac{\left(x-x_{i}\right)^{j-2}}{(j-2) !}\right|^{2} d x \\
\leq \cdots \leq\left(\frac{\delta^{2}}{\pi^{2}}\right)^{k} \int_{y_{i-1}}^{y_{i}}\left|f^{(k)}(x)-f^{(k)}\left(x_{i}\right)\right|^{2} d x \\
\leq\left(\frac{\delta^{2}}{\pi^{2}}\right)^{k+1} \int_{y_{i-1}}^{y_{i}}\left|f^{(k+1)}(x)\right|^{2} d x .
\end{aligned}
$$

Thus, for $f \in B^{2}(\Omega)$, invoking Bernstein's inequality $k+1$ times, one obtains

$$
\left\|f-A_{k} f\right\| \leq\left(\frac{\delta}{\pi}\right)^{k+1}\left\|f^{(k+1)}\right\| \leq\left(\frac{\delta \omega}{\pi}\right)^{k+1}\|f\| .
$$

For $\delta \omega<\pi$, Lemma 3 finishes the proof.

Remarks. 1. A statement for the stability is obtained as in Corollary 1. Setting $e_{i j}=j !^{-1} A_{k}^{-1} P\left(\left(x-x_{i}\right)^{j} \chi_{i}\right) \in B^{2}(\Omega)$ gives the series expansion

$$
f(x)=\sum_{i} \sum_{j=0}^{k} f^{(j)}\left(x_{i}\right) e_{i j}(x)
$$

similar to Corollary 2. 
2. The interest of Theorem 4 lies in the favorable speed of convergence. From regular sampling with derivatives [23, 24] the optimal sampling rate is known to be $\delta=(k+1) \pi / \omega$. With other Wirtinger-type inequalities [5], or using a good estimate for the remainder term of Taylor's polynomial, the estimate of the required density $\delta$ can be improved to $\delta \omega=O(k)$.

4.3. Irregular sampling in higher dimensions. Finally we look at the irregular sampling problem in higher dimensions. Although the numerical constants get worse with increasing dimension, we include a reconstruction algorithm because no constructive method for the complete reconstruction from random samples seems to be known in higher dimension. Partial results can be found in [25, 22, $3]$; for the qualitative reconstruction theory see $[9,10]$.

The notation is the same after interpreting all quantities as vectors in $R^{n}$ : $B^{2}(\Omega)=\left\{f \in L^{2}\left(R^{n}\right): \operatorname{supp} \hat{f} \subseteq \Omega\right\}$ is now a closed subspace of $L^{2}\left(R^{n}\right)$, where $\Omega \subseteq R^{n}$ is compact. The projection from $L^{2}\left(R^{n}\right)$ onto $B^{2}(\Omega)$ is $(P f)^{-}=$ $\chi_{\Omega} \widehat{\widehat{f}}$. The special extension of the spectrum $\Omega$ along the coordinate axes is measured by $\omega_{i}=\sup _{\mathrm{t}=\left(t_{1}, t_{2}, \ldots, t_{n}\right) \in \Omega}\left|t_{i}\right|$.

A sampling set $X=\left(x_{i}\right)_{i \in I}$ in $R^{n}$ is said to be $\delta=\left(\delta_{1}, \ldots, \delta_{n}\right)$-dense if $\bigcup_{i \in I} B_{\delta}\left(x_{i}\right)=R^{n}$, where $B_{\delta}(x)$ denotes the cube $\prod_{i=1}^{n}\left[\xi_{i}-\delta_{i} / 2, \xi_{i}+\delta_{i} / 2\right]$ centered at $x=\left(\xi_{1}, \ldots, \xi_{n}\right) \in R^{n}$. (Note that for dimension $n=1$, this definition differs slightly from the density used in the previous sections.) For every $\delta$-dense set $X$ a partition of unity $\left(\psi_{i}\right)_{i \in I}$ is chosen with the properties: $\operatorname{supp} \psi_{i} \subseteq B_{\delta}\left(x_{i}\right), 0 \leq \psi_{i}(x) \leq 1$, and $\sum_{i} \psi_{i}(x) \equiv 1$.

Theorem 5. Given $\Omega \subseteq R^{n}$ with linear extensions $\omega=\left(\omega_{1}, \ldots, \omega_{n}\right)$ and $B^{2}(\Omega)$, choose $\delta=\left(\bar{\delta}_{1}, \ldots, \delta_{n}\right)$ such that $\delta \cdot \omega=\sum_{i=1}^{n} \delta_{i} \omega_{i}<\ln 2$. If $X=\left(x_{i}\right)_{i \in I}$ is a $\delta$-dense sampling set in $R^{n}$, then every $f \in B^{2}(\Omega)$ can be reconstructed from its sampled values $f\left(x_{i}\right)$ by means of the following iteration procedure:

$$
\begin{gathered}
\phi_{0}=P\left(\sum_{i} f\left(x_{i}\right) \psi_{i}\right), \\
\phi_{n+1}=\phi_{n}-P\left(\sum_{i} \phi_{n}\left(x_{i}\right) \psi_{i}\right),
\end{gathered}
$$

and

$$
f=\sum_{n=0}^{\infty} \phi_{n}
$$

where all sums converge in $L^{2}\left(R^{n}\right)$.

Proof. We only have to show that the operator $A: B^{2}(\Omega) \rightarrow B^{2}(\Omega)$

$$
A f(x)=P\left(\sum_{i} f\left(x_{i}\right) \psi_{i}(x)\right)
$$

is invertible on $B^{2}(\Omega)$. As in $\S 3$, the $\delta$-oscillation of a function is defined by

$$
\operatorname{osc}_{\delta} f(x)=\sup _{\left|u_{i}\right| \leq \delta_{i}}|f(x+u)-f(x)| \text {. }
$$

Then 


$$
\begin{aligned}
\|f-A f\|^{2} & \leq \int_{R^{n}}\left|\sum_{i}\left(f(x)-f\left(x_{i}\right)\right) \psi_{i}(x)\right|^{2} d x \\
& \leq \int\left|\sum_{i} \operatorname{osc}_{\delta} f(x) \psi_{i}(x)\right|^{2} d x=\left\|\operatorname{osc}_{\delta} f\right\|^{2} .
\end{aligned}
$$

Since $f \in B^{2}(\Omega)$ is an entire function, its Taylor series expansion at $x$ can be used to obtain a pointwise estimate for the oscillation:

$$
\begin{aligned}
\operatorname{osc}_{\delta} f(x) & =\sup _{\left|u_{i}\right| \leq \delta_{i}}|f(x+u)-f(x)| \\
& =\sup _{\left|u_{i}\right| \leq \delta_{i}}\left|\sum_{|\alpha| \geq 1} \frac{u^{\alpha}}{\alpha !} D^{\alpha} f(x)\right| \leq \sum_{|\alpha| \geq 1} \frac{\delta^{\alpha}}{\alpha !}\left|D^{\alpha} f(x)\right|,
\end{aligned}
$$

where the multi-index notation $\alpha=\left(\alpha_{1}, \ldots, \alpha_{n}\right), u^{\alpha}=\prod_{i=1}^{n} u_{i}^{\alpha_{i}}, D^{\alpha} f(x)=$ $\partial^{|\alpha|} f\left(x_{1}, \ldots, x_{n}\right) / \partial^{\alpha_{1}} x_{1} \partial^{\alpha_{2}} x_{2} \cdots \partial^{\alpha_{n}} x_{n}, \alpha !=\alpha_{1} ! \alpha_{2} ! \cdots \alpha_{n} !$, and $|\alpha|=\alpha_{1}+\cdots+$ $\alpha_{n}$ has been used.

Consequently, one obtains

$$
\left\|\operatorname{osc}_{\delta} f\right\| \leq \sum_{|\alpha| \geq 1} \frac{\delta^{\alpha}}{\alpha !}\left\|D^{\alpha} f\right\| .
$$

By the multivariate version of Bernstein's inequality $\left\|D^{\alpha} f\right\| \leq \omega^{\alpha}\|f\|$ for $f \in$ $B^{2}(\Omega)$ one arrives at

$$
\left\|\operatorname{osc}_{\delta} f\right\| \leq\left(\sum_{|\alpha| \geq 1} \frac{\delta^{\alpha} \omega^{\alpha}}{\alpha !}\right)\|f\|=\left(e^{\delta \cdot \omega}-1\right)\|f\| .
$$

If $\delta \cdot \omega<\ln 2$, then $A$ is invertible on $B^{2}(\Omega)$ and the rest follows from Lemma 3.

Remarks. 1. Stability and series expansion are formulated exactly as in Corollaries 1 and 2.

2. If the spectrum is the cube $\Omega=\left[-\omega_{0}, \omega_{0}\right]^{n}$ and $\delta_{1}=\cdots=\delta_{n}=\delta_{0}$, then the density has to satisfy

$$
\delta_{0}<\frac{\ln 2}{n \omega_{0}}
$$

which gets worse with increasing dimension $n$.

\section{ACKNOWLEDGMENT}

I would like to thank the referee for his valuable suggestions which have helped to improve this paper.

\section{BIBLIOGRAPHY}

1. A. Beurling, Local harmonic analysis with some applications to differential operators, Some Recent Advances in the Basic Sciences, Belfer Grad. School of Science, Annual Science Conference Proc. (A. Gelbart, ed.), Vol. I, 1962-64, pp. 109-125.

2. A. Beurling and P. Malliavin, On the closure of characters and the zeros of entire functions, Acta Math. 118 (1967), 79-95.

3. P. L. Butzer and L. Hinsen, Two-dimensional nonuniform sampling expansions. I, II, Appl. Anal. 32 (1989), 53-68, 69-85. 
4. P. L. Butzer, W. Splettstößer, and R. Stens, The sampling theorem and linear prediction in signal analysis, Jahresber. Deutsch. Math.-Verein. 90 (1987), 1-70. .

5. W. J. Coles, A general Wirtinger-type inequality, Duke Math. J. 27 (1960), 133-138.

6. R. J. Duffin and A. C. Schaeffer, A class of nonharmonic Fourier series, Trans. Amer. Math. Soc. 72 (1952), 341-366.

7. Ky Fan, O. Taussky, and J. Todd, Discrete analogs of inequalities of Wirtinger, Monatsh. Math. 59 (1955), 73-90.

8. H. G. Feichtinger, Discretization of convolution and reconstruction of band-limited functions from irregular sampling, in Progress in Approximation Theory (P. Nevai and A. Pinkus, eds.), Academic Press, Boston, 1991, pp. 333 -345.

9. H. G. Feichtinger and K. Gröchenig, Iterative reconstruction of multivariate band-limited functions from irregular sampling values, SIAM J. Math. Anal. 23 (1992), 244-261.

10. __ Irregular sampling theorems and series expansions of band-limited functions, J. Math. Anal. Appl., July, 1992.

11. _ Multidimensional irregular sampling of band-limited functions in $L^{p}$-spaces, in $\mathbf{M u l -}$ tivariate Approximation Theory IV (C. K. Chui, W. Schempp and K. Zeller, eds.), ISNM 90, Birkhäuser, Basel, 1989, pp. 135-142.

12. __ Error analysis in regular and irregular sampling theory, to appear in Appl. Anal.

13. H. G. Feichtinger, K. Gröchenig, and M. Hermann, Iterative methods in irregular sampling theory, Numerical Results, vol. 7, Aachener Symposium für Signaltheorie, ASST 1990, Aachen, Informatik Fachber. 253, Springer, 1990, pp. 160-166.

14. K. Gröchenig, A new approach to irregular sampling of band-limited functions, NATO ASI "Harmonic Analysis and Applications" (Il Ciocco, Italy, 1989) (J. S. and J. L. Byrnes, eds.), Kluwer, Boston, 1990, pp. 251-260.

15. G. Hardy, J. E. Littlewood, and G. Pólya, Inequalities, 2nd ed., Cambridge Univ. Press, 1952.

16. A. J. Jerri, The Shannon sampling theorem-its various extensions and applications. A tutorial review, Proc. IEEE 65 (1977), 1565-1596.

17. H. Landau, Necessary density conditions for sampling and interpolation of certain entire functions, Acta Math. 117 (1967), 37-52.

18. __ Sampling data transmission and the Nyquist rate, Proc. IEEE 55 (1967), 1701-1706.

19. N. Levinson, Gap and density theorems, Colloq. Publ., vol. 26. Amer. Math. Soc., Providence, RI, 1940.

20. F. A. Marvasti, $A$ unified approach to zero-crossing and nonuniform sampling of single and multidimensional systems, Nonuniform (P. O. Box 1505, Oak Park, IL 60304), 1987.

21. F. Marvasti and M. Analoui, Recovery of signals from nonuniform samples using iterative methods, Proc. Internat. Sympos. Circuits Systems, Portland, OR, May 1989.

22. F. A. Marvasti, An iterative method to compensate for the interpolation distortion, IEEE Trans. Acoust. Speech Signal Process. 37 (1989), 1619-1621.

23. A. Papoulis, Signal analysis, McGraw-Hill, New York, 1977.

24. M. D. Rawn, A stable nonuniform sampling expansion involving derivatives, IEEE Trans. Inform. Theory 36 (1989), 1223-1227.

25. K. D. Sauer and J. P. Allebach, Iterative reconstruction of band-limited images from nonuniformly spaced samples, IEEE Trans. Circuits and Systems 34 (1987), 1497-1506.

26. R. G. Wiley, Recovery of band-limited signals from unequally spaced samples, IEEE Trans. Comm. 26 (1978), 135-137.

27. S. Yeh and H. Stark, Iterative and one-step reconstruction from nonuniform samples by convex projections, J. Opt. Soc. Amer. A 7 (1990), 491-499.

28. D. C. Youla and H. Webb, Image restoration by the method of convex projections: Part I, IEEE Trans. Med. Imag. 1 (1982), 81-94.

29. R. Young, An introduction to nonharmonic Fourier series, Academic Press, New York, 1988.

Department of Mathematics U-9, University of Connecticut, Storrs, Connecticut 06269-3009

E-mail address: groch@uconnvm.bitnet 